Open Access

\title{
Interoperability technology of sports health monitoring equipment based on multi- sensor information fusion
}

\author{
Di Zhang
}

\author{
Correspondence: zd17209481@163. \\ com \\ Department of Physical Education, \\ Northeastern University, Shenyang \\ 110819, Liaoning, China
}

\begin{abstract}
Nowadays, the pace of society is accelerating, people's living standard has been greatly improved compared with the past, and we pay more and more attention to the real-time, flexibility, and intelligence of medical services. Information fusion technology is the study of how to effectively and comprehensively utilize multisensor information to overcome information imperfections and uncertainties. In recent years, it has become a hotspot for research, but with the development of communication technology, electronic technology, and computers, the form of information becomes complicated and diverse in science and technology such as technology. In this case, the information obtained by a single sensor is always imperfect, so multiple sensors must be used to detect the target from multiple angles. Obtaining more dimensional information about the target and fusing it to ensure the integrity and accuracy of the information helps to accurately determine the physiological health of the human body. This paper analyzes the multi-sensor information fusion technology in detail, discusses the basic principle of multi-sensor information fusion, fusion process, fusion level, architecture and general fusion algorithm, constructs a sensor information fusion technology and its application model in biomedical detection, from the application framework, control structure of multi-sensor integrated system, information fusion, etc. The current situation of multi-sensor information fusion technology is studied in several aspects. An interoperability technology of sports health monitoring equipment based on multisensor information fusion is constructed. The experimental results show that the experimental efficiency is improved by $20 \%$ after using the application model in this paper, which has certain practicability.
\end{abstract}

Keywords: Sensor information fusion technology, Biomedical detection, Fusion algorithm, Multi-sensor integrated system, Biological testing (c) The Author(s). 2021 Open Access This article is licensed under a Creative Commons Attribution 4.0 International License, which permits use, sharing, adaptation, distribution and reproduction in any medium or format, as long as you give appropriate credit to the original author(s) and the source, provide a link to the Creative Commons licence, and indicate if changes were made. The images or other third party material in this article are included in the article's Creative Commons licence, unless indicated otherwise in a credit line to the material. If material is not included in the article's Creative Commons licence and your intended use is not permitted by statutory regulation or exceeds the permitted use, you will need to obtain permission directly from the copyright holder. To view a copy of this licence, visit http://creativecommons.org/licenses/by/4.0/. 


\section{Introduction}

\subsection{The necessity of application of sensor information fusion technology in biomedical detection}

The function of sensor in application is just like the perception organ of the human body, through which we can obtain all kinds of important information we want to obtain. Multi-sensor information fusion technology can be used for data fusion of homogeneous sensors and heterogeneous sensors, so it can obtain more information resources than single sensor, making the information after fusion more reliable, more comprehensive, and more reference value. It solves the shortcomings of single sensors in these issues and avoids bottlenecks in the information blind area of single sensor technology. Not only will you get better information processing results but also the entire decision process will be more accurate and your final decision will be closer to the real situation. Note that the sensors highlighted here are not a narrow concept, but a more general and comprehensive system that can capture all sorts of effective information. Similarly, the integration mentioned here is not a narrow concept. This means using different sensors to work together to collect, process, analyze, integrate different information, and effectively fuse different pieces of information. At present, the socalled information processing technology has become a key link in the field of information acquisition and processing. Therefore, the rapid development of pre-information technology has become a key frontier technology, and has received great attention in the research.

\subsection{Literature review}

Human body sensors can be implanted or attached to the human body to monitor the physiological parameters of patients. The incorrect data caused by sensor failure or incorrect placement on the human body will seriously affect the diagnosis of clinicians. Therefore, the detection of sensor data failure has been widely studied in recent years. In the medical field, most of the typical sensor fault detection methods ignore the fact that the physiological indicators of patients will not change synchronously, and it is difficult to determine the real fault due to the mixture of fault value caused by disease and abnormal physiological data. Based on these facts, Yang Y proposed a data fault detection mechanism in medical sensor network [1]. The mechanism includes using dynamic local outlier factor (d-lof) algorithm to identify outlier sensing data vector, using linear regression model based on trapezoidal fuzzy number to predict which readings in peripheral data vector are suspected to be wrong, and a new fault state judgment criterion is proposed according to the predicted values. With the rapid development of wireless communication technology and semiconductor technology, wireless sensor network (WSN) has been widely used in many applications [2]. As an application of WSN, wireless medical sensor network (WMSN) can improve the medical quality, and has become more and more important in modern medical system. In WMSN, physiological data is collected by sensors deployed in patients and sent to medical professionals' mobile devices via wireless communication. Health professionals can access the status of patients anytime and anywhere [3]. Data breaches can compromise patient privacy, and malicious changes can compromise patient health. He D proposes a reliable anonymous authentication protocol for healthcare applications using WMSN. 
Compared to Kumar's protocol, this protocol has stronger security and computational efficiency. Therefore, it is suitable for healthcare applications that use WMSN. With the development and maturity of wireless communication technology, wireless sensor networks have been widely used in various environments to obtain specific information. Wireless medical sensor networks (WMSN) are gaining increasing attention as a specialized application of wireless sensor networks in healthcare, as they have the potential to improve the quality of medical services, which are then accessed by healthcare professionals using mobile devices $[4,5]$.

Due to the openness of wireless communication, how to ensure secure communication has become an important issue. Because medical sensor network (BSN) is usually limited in resources and vulnerable to environmental impact and malicious attacks, sensor data errors will inevitably occur, which may lead to false alarms, medical diagnosis errors, and even serious misjudgments. Therefore, before using it in medical diagnosis, it is necessary to detect and delete the wrong sensory data as much as possible. Most of the available work directly adopts the fault detection scheme developed in traditional wireless sensor network (WSN) for human body sensor fault detection. However, BSN uses a very limited number of sensors to collect important information, but lacks the information redundancy provided by the densely deployed sensor nodes in traditional WSN. In view of this, Zhang $\mathrm{H}$ proposed a sensor fault detection scheme based on Bayesian network model, which relies on historical training data to establish the conditional probability distribution of human body sensor readings, rather than the redundant information collected from a large number of sensors.

\section{Interoperability technology of sports health monitoring equipment based on multi-sensor information fusion}

\subsection{Sensor information fusion technology}

Data layer fusion is to use multiple sensors to collect the information of the target to be measured, then directly fuse the original data, extract the target feature vector from the fusion information, and finally obtain the recognition results according to the feature vector information. In the data layer fusion, the sensor detects the same characteristics of the target to be measured, that is to say, the acquired data are homogeneous. Data layer fusion is not suitable for heterogeneous data, which is also the limitation of data layer fusion [6].

Data layer fusion is the fusion of the original data collected by sensors, that is, the fused data has the greatest authenticity and comprehensiveness. Therefore, the results of data layer fusion are more accurate than those of feature level fusion and decision level fusion. However, data layer fusion also has its own limitations, which are mainly reflected in the following aspects: data layer fusion faces a large number of original data, sometimes it can be said to be massive, which will inevitably increase the amount of calculation $[7,8]$. The number of fusions is greatly increased. The fusion takes a long time, and the real-time performance of the system cannot be guaranteed. Since most of the original data detected by sensors are incomplete and uncertain, it is necessary for the fusion system to have good robustness and error correction ability to obtain highprecision target discrimination. Data layer fusion can only be performed for homogeneous data, but not for heterogeneous data. Due to the large amount of data detected 
by sensors, the fusion system cannot distinguish all interference information and cannot guarantee that each data is reliable. Therefore, it is necessary to enhance the antiinterference performance. Data layer fusion is usually used for multi-source image synthesis and direct synthesis of detection signal waveform. This fusion mode is also called pixel level fusion $[9,10]$. Weight refers to the relative importance of a factor in the overall evaluation. Carrier frequency refers to the frequency used to load a signal onto a wave of a fixed frequency. Pulse width refers to the duration of the pulse that can reach the maximum value in the electronic field.

\subsection{Biomedical testing}

The biosensor is based on the change of signal generated by the reaction between the detected substance and the detection reagent. It has attracted people's attention because of its fast, sensitive, accurate, and high selectivity in the process of biological detection. Several common biosensors are briefly introduced. The biosensor based on reflectance spectrum is the most widely studied and effective detection method. It is mainly based on the principle of thin film interference $[11,12]$. When the refractive index of the substrate is affected by the analysis object, the reflection spectrum will move, so that the analyte can be detected quantitatively according to the reflection spectrum offset. The biosensor based on fluorescence spectrum makes use of the photophysical properties of the fluorescent group in the biosensor, since this is affected by the analysis target, the output form of the fluorescence signal changes, such as the shift and fluctuation of the fluorescence peak position. Fluorescent biosensors are a combination of research in photophysical chemistry and synthetic organic chemistry. Due to the high sensitivity of fluorescence analysis, this type of sensor has attracted people's attention. Surface plasmon resonance biosensor has the advantages of no damage, no label, and real-time detection of biomolecule dynamic reaction process and high sensitivity. It has become a new technology with rapid development in recent years, and it is also one of the research hotspots in the application field of sensor detection [13]. SPR is a physical optical phenomenon. SPR sensor mainly uses the surface enrichment effect of metal nanoparticles and local surface plasmon resonance effect to improve the detection sensitivity of biosensor. As SPR is very sensitive to the refractive index of metal surface dielectric, the response strength of SPR will be different as long as the refractive index of surface dielectric changes.

\subsection{Biological index detection method}

The detection method used in this paper is to use the change of reflection spectrum signal of porous silicon as the sensing signal of biomolecule or chemical composition. In other words, the recognition and detection of biomolecules by porous optical biosensor is mainly based on the change of refractive index signal before and after the coupling of porous silicon and biomolecules. In the range of visible light or longer wavelength, the aperture of porous silicon layer is much smaller than the wavelength of incident light [11].

$$
\begin{aligned}
& (1-\rho) \frac{n_{s i}^{2}-n_{e f f}^{2}}{n_{s i}^{2}+2 n_{e f f}^{2}}+\rho \frac{n_{a i r}^{2}-n_{e f f}^{2}}{n_{s i}^{2}+2 n_{e f f}^{2}}=0 \\
& f(x)=\frac{1}{N h} \sum_{i=1}^{N} k\left(\frac{X_{i}-x}{h}\right)
\end{aligned}
$$




$$
k(x)=\frac{1}{\sqrt{2 \pi}} \exp \left(-\frac{x^{2}}{2}\right)
$$

When porous silicon is used as a sensor for detecting gas or solution, the gas or liquid diffuses into the pores of porous silicon [14]. When the volume fraction of gas or liquid in porous silicon is $V(0<V<\rho)$ and constant, the porous silicon layer will become a mixture of air, silicon, and gas or liquid molecules, and its effective refractive index satisfies the following conditions:

$$
\begin{aligned}
& (1-\rho) \frac{n_{s i}^{2}-n_{e f f}^{2}}{n_{s i}^{2}+2 n_{e f f}^{2}}+(\rho-v) \frac{n_{\text {air }}^{2}-n_{e f f}^{2}}{n_{\text {air }}^{2}+2 n_{\text {eff }}^{2}}+v \frac{n_{n}^{2}-n_{e f f}^{2}}{n_{n}^{2}+2 n_{e f f}^{2}}=0 \\
& h_{t}=\tanh \left(w_{c} x_{t}+u_{u}\left(r_{t} \Theta h_{t-1}\right)+b_{c}\right) \\
& h_{t}=z_{t} \Theta h_{t-1}+\left(1-z_{t}\right) \Theta h_{t}
\end{aligned}
$$

The film interference formula is obtained from the above formula:

$$
\begin{aligned}
& 2 \mathrm{~d}\left(\sqrt{\mathrm{n}_{\text {eff }}^{2}-n_{\text {air }}^{2}} \sin ^{2} i\right)=m \lambda \\
& 2 n_{e_{f f}} d=m \lambda \\
& \mathrm{E}_{\mathrm{j}} \frac{\frac{1}{2 u_{j}} \sum_{i=1}^{n_{j}} \sum_{r=1}^{n_{j}}\left|y_{j i}-y_{j r}\right|}{n_{j}^{2}}
\end{aligned}
$$

It can be seen from the above formula that when the effective refractive index changes, the position of the interference peak will move $[15,16]$. Therefore, the number of biomolecules entering the porous silicon layer can be determined by the relationship between the effective refractive index and the position shift of the interference peak, so as to realize the sensitivity calculation of the porous silicon biosensor and realize the detection of biological indicators.

\subsection{Multi-sensor information fusion algorithm}

\subsubsection{Weighted average method}

Weighted average method is a real-time processing fusion algorithm. Its essence is to process the information from multiple sensors in the future, and then weighted average the weight of a single sensor to obtain the final fusion result. This method is suitable for obtaining fusion value in dynamic environment, and the difficulty lies in solving the weight problem of single sensor [17]. Firstly, the algorithm analyzes the dataset and obtains the information of each dimension, including the span of each dimension. Analyze the recordset dataset to obtain the minimum and maximum values of each dimension in the recordset. In order to promote grid clustering, the maximum and minimum values need to be modified. The maximum and minimum values of each dimension are corrected according to the following formula.

$$
\begin{aligned}
\min _{i} & =\min _{i}-k \times \operatorname{steplen}(i) \\
\max _{i} & =\max _{i}+k \times \operatorname{steplen}(i) \\
u_{(j / i)} & =w_{i j} A_{i}
\end{aligned}
$$




$$
s_{j}=\sum_{i} c_{i j} u_{(j / i)}
$$

\subsubsection{Bayesian reasoning}

This method is suitable for data fusion in non-dynamic environment. It uses probability distribution to express information. In this method, each node in the sensor system is regarded as Bayesian estimation [18, 19]. When there is certain evidence (measure), Bayesian reasoning can determine the probability of hypothetical events according to the evidence (measurement); when there is new evidence (measure), the probability function of the assumed event can be updated by using the probability of new evidence and the likelihood function before generating new evidence. Finally, the system decision is given by some criteria. Bayesian network reasoning is a tool for decision support and causal discovery when information is incomplete. It is based on the probability distribution and considers that the values of all variables are controlled by the probability distribution [20]. Based on the observed data, the correct decisions can be made by calculating these probabilities. Because it provides a quantitative hypothesis method based on evidence support, it not only provides a theoretical basis for the algorithm of direct manipulation probability but also provides a theoretical framework for the analysis of algorithms without explicit probability calculation formula. Therefore, probability inference in Bayesian learning plays an important role in machine learning. The core of Bayesian network reasoning is to calculate the posterior conditional probability distribution [21,22]. If the set of all variables is $x$, the set of evidence variables is $e$, and the set of query variables is $Q$, then the task of Bayesian network reasoning is to calculate the conditional probability distribution under the given set of evidence variables [23]. It can be formally described as:

$$
\begin{aligned}
& p(Q \mid E=e)=\frac{p(Q, E=e)}{p(E=e)} \\
& y=(D a \times D b)-1 \\
& E=\frac{\sum_{j=1}^{k} \sum_{h=1}^{k} \sum_{t=1}^{n_{j}} \sum_{r=1}^{n_{h}}\left|y_{i j}-y_{h r}\right|}{2 n^{2} u} \\
& E w=\sum_{j=1}^{k} G_{i j} P_{j} s_{j}
\end{aligned}
$$

\subsubsection{Clustering analysis}

Clustering analysis is a heuristic algorithm, which mainly clusters data according to some similarity criteria, and decomposes each data group into corresponding target categories [24]. This method is widely used in target recognition, and the number of clusters is not clear.

\subsection{Interoperable access control technology}

For a large-scale distributed system, divide and conquer management is usually adopted, which leads to the concept of "domain," that is, the system blocks constitute the "domain." The traditional access control models, such as autonomous access control model, mandatory access control model, or role-based access control model, are 
mainly used in centralized systems and cannot meet the requirements of inter domain interoperability access control. For the collaborative work environment composed of multiple distributed and heterogeneous security domains (autonomous domains), multi domain security interoperability provides a secure and effective way to realize data interaction and resource sharing among domains.

Security domain is a bounded area composed of protected objects and user groups, and a set of security policies is managed and maintained by security administrators. Each domain can use different security models, classification patterns, syntax, and constraints to express its own information security policies, and has the right to allow legitimate requests and prevent illegal access. Security domain is also called autonomous domain.

Multi domain is composed of multiple security domains with mutual contract relationship. When a security domain needs to allow unknown entities or users to access local resources, corresponding security mechanisms must be set up to ensure that the access can be controlled within the scope of pre-defined known sharing policies. When such cross domain access is allowed between security domains, and each domain still uses the local original security policy, it can be regarded as a multi domain environment composed of these security domains:

$$
\begin{aligned}
& \mathrm{V}_{\mathrm{a}}=\sum_{j=2}^{k} \sum_{h=1}^{j-1} G_{j h}\left(P_{j} s_{h}+P_{h} s_{j}\right) D_{j h} \\
& y_{t}=\sum_{j=2}^{k} \sum_{h=1}^{j-1} G_{j h}\left(p_{j} s_{h}+p_{h} s_{j}\right) D_{j h}\left(1-D_{j h}\right)
\end{aligned}
$$

There are two main characteristics of interoperable access control:

1) Distributed management: every independent distributed application has its own security policy, so it is difficult to realize access control in a unified and centralized way:

$$
\begin{aligned}
& \ln \left(\frac{F I_{i t}}{F I_{i t}-1}\right) * k_{t 1}[i]=\sum_{j} \cos \left(w_{i}^{1}, w_{j}^{2}\right)+\alpha+\beta \operatorname{In} F I_{i t}-1+\phi X_{i t}-1+v_{i}+\tau_{t} \\
& \theta=-\frac{1}{T} \operatorname{In}(1+\beta) \\
& \sigma_{\mathrm{ikjl}}= \begin{cases}\frac{\mathrm{n}}{\Delta_{\mathrm{ikjl}}} \sqrt{\sum_{\mathrm{s}=1}^{\mathrm{n}}\left(\mathrm{x}_{\mathrm{ik}}(\varepsilon)-\mathrm{x}_{\mathrm{jl}}(\varepsilon)\right)^{2} \Delta_{\mathrm{ikjl}}(\varepsilon)} & \Delta_{\mathrm{ikjl}}>0 \\
0 & \Delta_{\mathrm{ikjl}}<0\end{cases} \\
& \mathrm{X}_{1}=\left\{\mathrm{X}_{1}(\mathrm{t}-4 \Delta \mathrm{t}), \mathrm{X}_{1}(\mathrm{t}-3 \Delta \mathrm{t}), \mathrm{X}_{1}(\mathrm{t}-2 \Delta \mathrm{t}), \mathrm{X}_{1}(\mathrm{t}-\Delta \mathrm{t})\right\}
\end{aligned}
$$

2) Cross domain access control: if we want to achieve access control in distributed environment, we must cooperate among the security domains formed by independent distributed applications to decide whether to grant access to local resources to users in the external domain. 
In multi-tier applications, the security of each domain becomes the primary problem of multi domain interoperability. Therefore, the access control of secure interoperability is mainly based on the following two principles:

1) Autonomy principle: if an access is allowed in a single domain system, the access is also allowed in the case of multi domain interoperability:

$$
\begin{aligned}
& \mathrm{Y}_{1}=\left\{\mathrm{Y}_{1,1}, \mathrm{Y}_{1,2}, \mathrm{Y}_{1,3}, \mathrm{Y}_{1,4}, \mathrm{Y}_{1,5}\right\} \\
& y_{i}=\beta\left(u_{i}, v_{i}\right)+\sum_{j=1}^{p} \beta_{j}\left(u_{i}, v_{i}\right) x_{i j}+\varepsilon_{j} \beta_{j}
\end{aligned}
$$

2) Security principle: if an access is forbidden in a single domain system, the access is also prohibited in the case of multi domain interoperability. These two principles are formally described as follows:

$A: U, R, P$, and $D$ represent user set, role set, permission set and security domain set of multi domain respectively;

$B: U_{D}, R_{D}$, and $\mathrm{P}_{\mathrm{D}}$ are recorded as $u, R, P$ in security domain $D$, respectively;

$C: d_{1}, d_{2}, d_{3}, \ldots d_{n}$ represent the domains in the multi domain security interoperability.

So, $\forall P \in P_{d_{i}}(i=1,2, \ldots n) \Rightarrow P \in P_{D}, \quad \forall P \notin P_{d_{i}}(i=1,2, \ldots n) \Rightarrow P \notin P_{D}$.

$$
\begin{aligned}
& \mathrm{Y}_{2}=\left\{\mathrm{Y}_{2,1}, \mathrm{Y}_{2,2}, \mathrm{Y}_{2,3}, \mathrm{Y}_{2,4}, \mathrm{Y}_{2,5}\right\} \\
& \mathrm{w}_{\mathrm{G}}{ }^{\mathrm{A}_{\mathrm{i}} \mathrm{A}_{j}}=\max \left\{0, W_{G} \cdot \varepsilon\left(f_{G}{ }^{A_{i}}, f_{G}{ }^{A_{j}}\right)\right\}
\end{aligned}
$$

\section{Experiments}

\subsection{Experimental data set}

The mHealth (mobile health) data set includes physical movement and vital signs recorded by 10 volunteers during multiple physical activities. Sensors placed on the subject's chest, right wrist, and left ankle are used to measure the motion experienced by different parts of the body, that is, acceleration, rate of rotation, and direction of the magnetic field. The sensor located in the chest also provides 2-lead ECG measurement, which can be used for basic heart monitoring, checking various arrhythmias, or checking the effect of exercise on ECG.

An additional fixed threshold is added to the relative error event trigger mechanism, which is an event trigger mechanism that combines relative error and absolute error. This additional threshold ensures that each node has a minimum event interval, and models the ETM system as an impulse system, which is conducive to describing the behavior of the system. This system structure provides stability guarantees for the event trigger mechanism. 


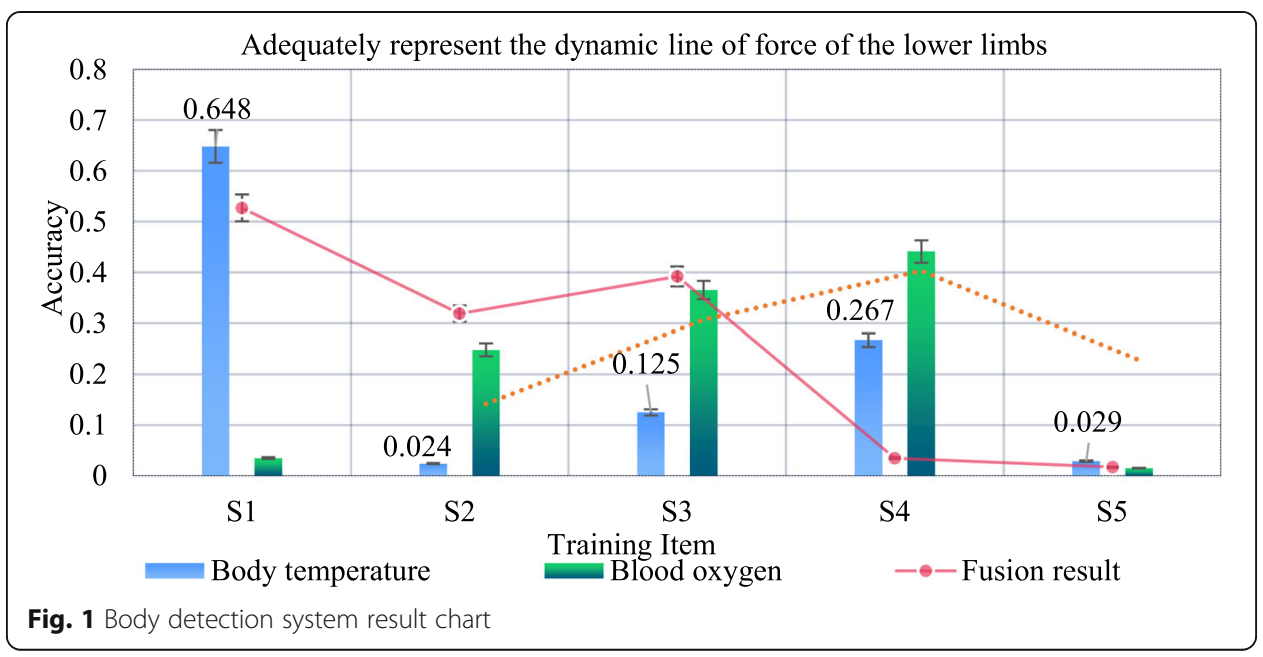

\subsection{Specific application steps}

1) For ECG signal measurement: the hardware level is mainly to complete the ECG amplifier circuit and ADC acquisition circuit design. The emphasis of digital filtering is to filter out $50 \mathrm{~Hz}$ power frequency interference and baseline drift. Finally, the ECG voltage data of 200 points per second is obtained. Used CT and MRI as test tools for the experiment.

2) Wireless sending and receiving data: the sending function is to send respiratory or ECG data wirelessly. The receiving function is to recognize the user instructions received from the wireless port, so as to switch the ECG/respiratory measurement function.

3) Pretreatment of experimental data: the amplitude of analog sensor signal is easily affected by interference source, so the system cannot directly use the original information. It is necessary to transform and process it, compensate for its zero drift, shape, filtering, amplification, sampling and a/D conversion of processed information, so as to convert analog quantity into digital quantity for output and use of upper equipment.

4) Local decision processing of information layer: in the continuous collection of data, the amount of data is very large, so we should deal with the data information at the bottom to make local decision, so as to reduce the pressure of data processing at the upper level.

5) The realization of feature layer of information fusion system: when there is abnormal data in the information layer, the feature layer needs to extract features from the basic data. The realization of the feature layer of fusion system is to extract the features of the transmitted data in the information layer, fuse the extracted feature information according to a certain algorithm, and get the fusion result.

\subsection{Data analysis design}

This is the era of big data. Data are the most reliable and direct source of analysis. Data classification and analysis is essential in the Internet of things system, so we can master the whole system and provide important data support for us to better design system functions [25]. The multi-sensor information fusion algorithm combines the 
Table 1 Fusion results of body temperature and blood oxygen

\begin{tabular}{llll}
\hline Sequence & Body temperature & Blood oxygen & Fusion result \\
\hline S1 & 0.648 & 0.035 & 0.527 \\
S2 & 0.024 & 0.248 & 0.319 \\
S3 & 0.125 & 0.365 & 0.392 \\
S4 & 0.267 & 0.441 & 0.035 \\
S5 & 0.029 & 0.015 & 0.017 \\
\hline
\end{tabular}

corresponding fusion algorithm to comprehensively and completely reflect the target object and environment information obtained by multiple sensors [26, 27]. In the whole multi-sensor information system, the information we collect is various, sometimes the information is complex. Therefore, it is required that the information fusion method we adopt has the corresponding processing ability, that is, it is not only robust but also parallel. In addition, there are higher requirements for the coordination ability of different monitoring methods and technologies, the operation speed and accuracy of the algorithm, and the interface performance of the information recognition system and the preprocessing system. Information plays an important role in the whole system.

\section{Results and discussion}

\subsection{Analysis of simulation data processing results}

As shown in Fig. 1, this paper based on the experimental data set. The assignment of the basic probability assignment function of three sensors is described. There were temperature, oxygen, and pulse rate sensors in the experiment, which were divided into groups A and group B. Through the probability distribution function calculated by multi-sensor information fusion algorithm, the fusion result of body temperature and blood oxygen fusion is obtained. Table 1 is a table of the effect of body temperature and blood oxygen combined fusion after collecting the sample, and the value is used to replace the degree of fusion.

As shown in Tables 1 and 2, for the algorithms mentioned in the second part, we regard them as the first level (the core algorithm of the local fusion center) and the second level (the core algorithm of the global fusion center) in the fusion system. Then the temperature, blood oxygen, and pulse rate of human body in normal indoor environment are monitored, and the health of people is judged according to these parameters.

It can be seen from Fig. 2, the fusion results that $\mathrm{S} 1=$ \{normal body temperature $36.2{ }^{\circ} \mathrm{C} \sim 37.3{ }^{\circ} \mathrm{C}$ normal blood oxygen $93-95 \%$ normal pulse rate 70 beats/min-80

Table 2 Fusion results of body temperature and pulse rate

\begin{tabular}{llll}
\hline Sequence & Body temperature & Pulse rate & Fusion result \\
\hline S1 & 0.648 & 0.003 & 0.235 \\
S2 & 0.024 & 0.165 & 0.326 \\
S3 & 0.125 & 0.327 & 0.169 \\
S4 & 0.267 & 0.042 & 0.027 \\
S5 & 0.029 & 0.025 & 0.164 \\
\hline
\end{tabular}




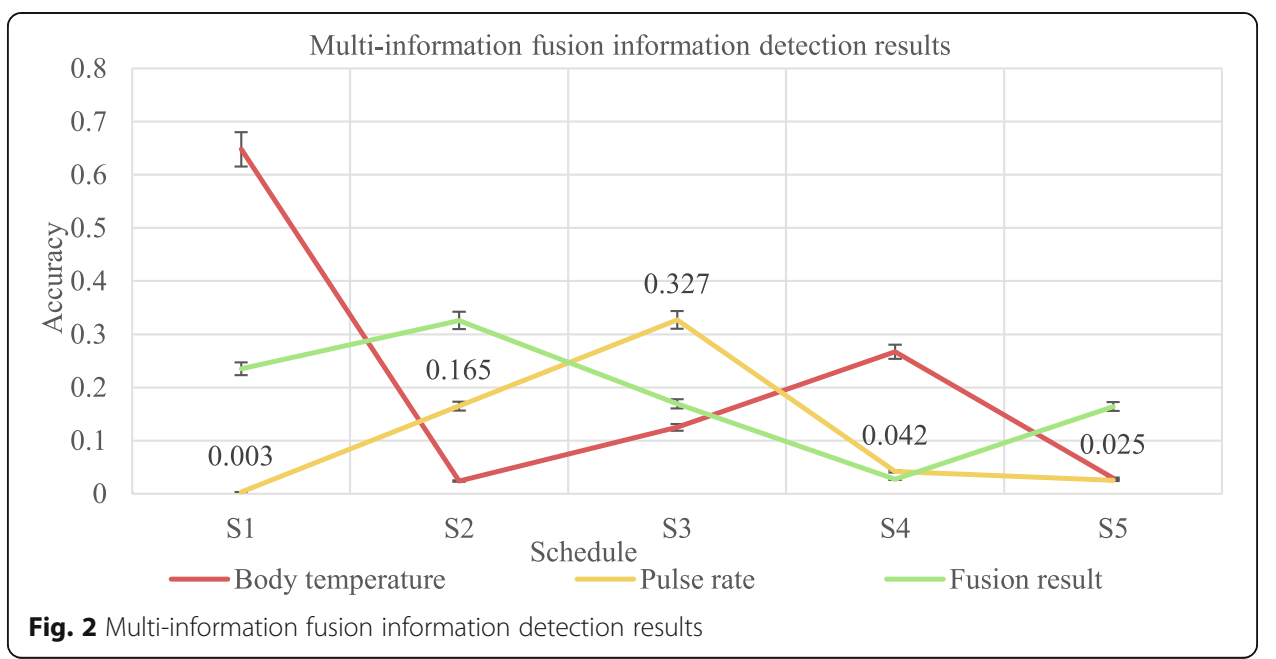

beats/min\} has the highest reliability. Multiple evidences support S1. Therefore, the fusion result is that the body temperature and blood oxygen are normal at this time.

\subsection{Efficiency analysis of application algorithm}

As shown in Fig. 3, the three algorithms are divided into a, B, and C. The three algorithms are weighted average method, Bayesian inference algorithm and cluster analysis method. The results of the three algorithms are shown in the figure. The simulation results show that the simulation results of the three algorithms can eliminate the jamming target, and the trajectory of the jamming target is not taken as the correlation result, and there is no serious deviation at the intersection. The target state feature weight, carrier frequency, pulse repetition period, and pulse width are $0.356,0.247$, and 0.198 respectively.

As shown in Fig. 4, the average absolute error between the results of the three algorithms and the target ideal value and the average single operation time of the three algorithms are listed in the figure. These three algorithms can be used to correlate the measurement data well. These three algorithms are based on updating the target state

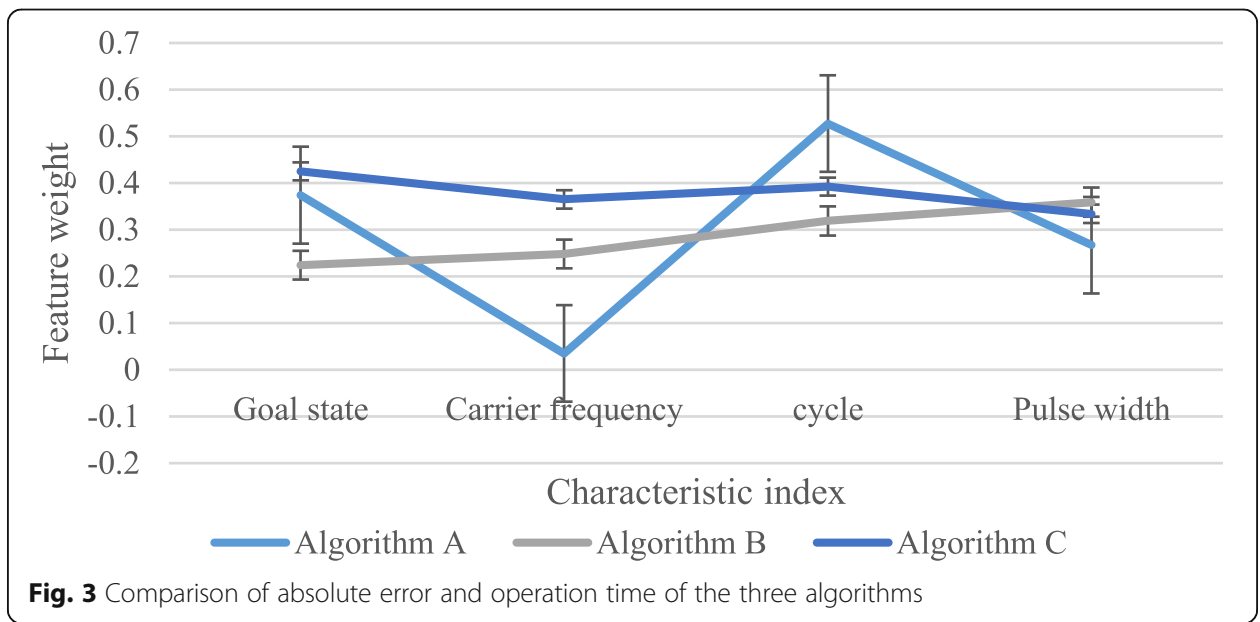




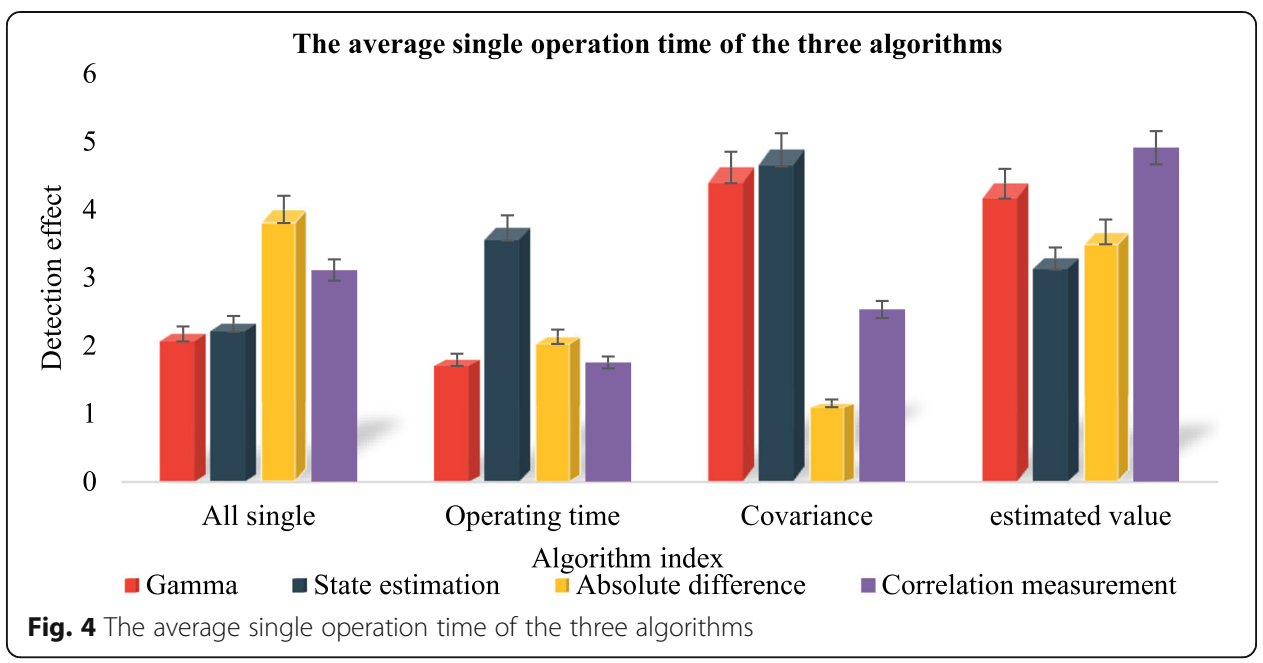

using association probability. The three algorithms calculate the association probability of each measurement value with the reference target in different ways, then update the state, and finally obtain the state estimation value.

As shown in Table 3, the difference between the calculated value and the covariance estimation value is that the correlation probability of the algorithm is calculated by the cross-correlation matrix and the posterior probability of the joint event. The gray correlation coefficient is obtained by calculating the absolute difference of feature information, and then the correlation probability is obtained. In terms of accuracy, with the decrease of signal-to-noise ratio, the accuracy of each algorithm decreases; with the increase of threshold value, the accuracy of each algorithm also decreases. Under the same SNR and the same threshold, the accuracy of algorithm a and algorithm B is far lower than that of algorithm $\mathrm{C}$, and the algorithm has the highest accuracy.

As shown in Fig. 5, the anti-jamming ability of weighted average method is weak, and it is easy to cause false correlation. This algorithm is only suitable for single target data association and is not practical. The result of the algorithm is very close to the ideal value of the target, and the error is very small. However, the algorithm only depends on the measured data to calculate the cross-correlation matrix. In practical application, the model is difficult to understand, the calculation is complex, the amount of calculation is large, and the calculation time is long.

As shown in Fig. 6, these three algorithms can be used to correlate survey data well. These three algorithms are based on updating the target state using association probability. The three algorithms calculate the association probability of each measurement value with the reference target in different ways, then update the state, and finally obtain the state estimation value.

Table 3 The cross-correlation matrix and the posterior probability

\begin{tabular}{lllll}
\hline Item & Gamma & State estimation & Absolute difference & Correlation measurement \\
\hline All single & 2.17 & 2.32 & 4 & 3.11 \\
Operating time & 1.79 & 3.73 & 2.13 & 1.75 \\
Covariance & 4.62 & 4.88 & 1.15 & 2.53 \\
Estimated value & 4.38 & 3.28 & 3.67 & 4.91 \\
\hline
\end{tabular}




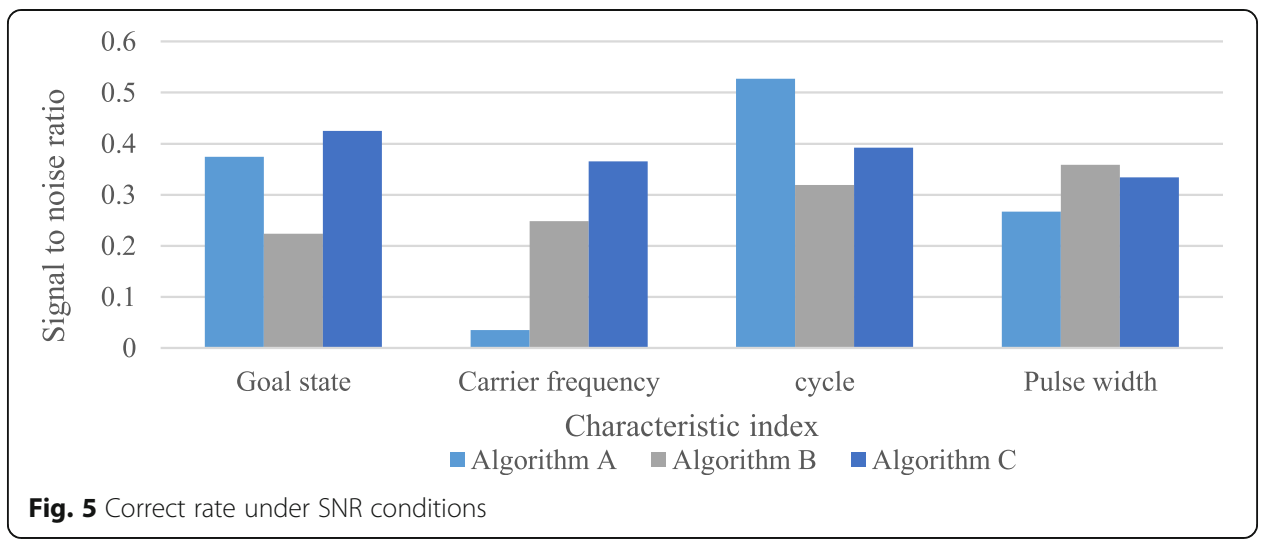

As shown in Table 4, the difference between the calculated value and the covariance estimation value is that the correlation probability of the algorithm is calculated by the cross-correlation matrix and the posterior probability of the joint event.

\subsection{Accuracy analysis of data fusion}

The mean square deviation between the fusion results of the three algorithms and the ideal angle is compared. The mean square error of the sensor is added to the original data provided by the sensor and the ideal angle value. After fusion, the fusion accuracy and performance of the sensor are improved, and the noise is suppressed to a certain extent, which shows the effectiveness of the three fusion algorithms. In the experiment, the clustering analysis method is very simple, but only suitable for the original data fusion.

As shown in Fig. 7, only the performance of fusion algorithm in fusion module is considered. The performance of clustering analysis algorithm is similar to the improved weighted fusion algorithm, but the clustering analysis method needs training samples, which is not suitable for the small number of training samples. The mean square error of the improved weighted fusion algorithm and equal weight fusion algorithm is relatively small, because the filtering processing is carried out before the fusion processing, thus reducing the sensor measurement deviation.

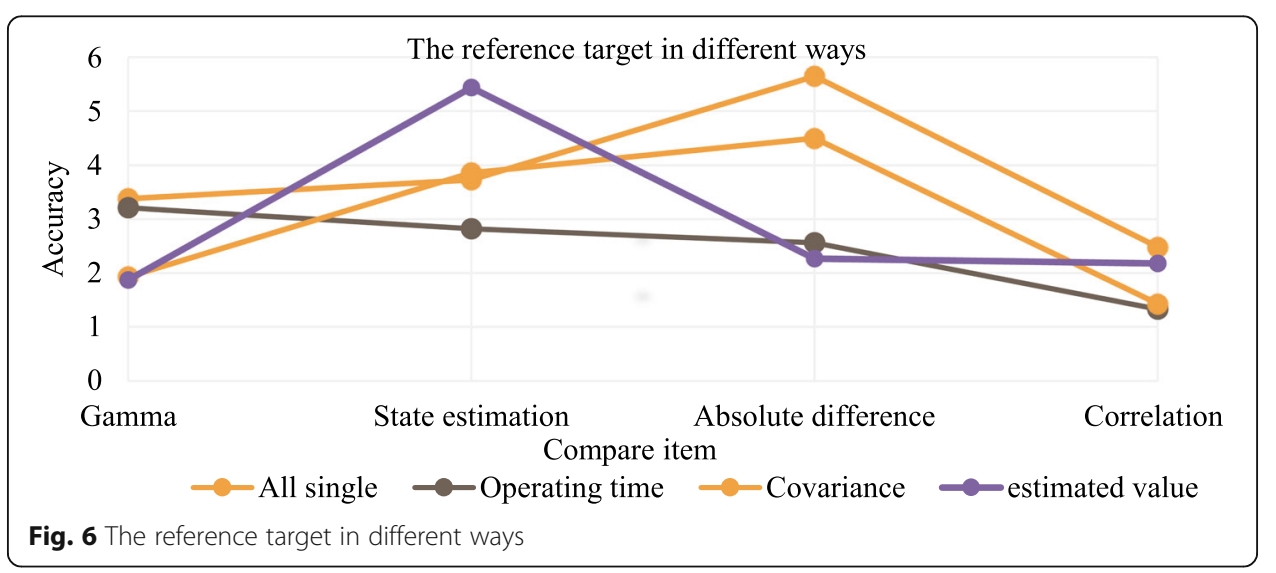


Table 4 The correlation probability of the algorithm

\begin{tabular}{lllll}
\hline Item & All single & Operating time & Covariance & Estimated value \\
\hline Gamma & 3.38 & 3.21 & 1.93 & 1.87 \\
State estimation & 3.73 & 2.82 & 3.86 & 5.44 \\
Absolute difference & 5.65 & 2.56 & 4.5 & 2.27 \\
Correlation & 2.48 & 1.33 & 1.43 & 2.18 \\
\hline
\end{tabular}

As shown in Fig. 8, when the performance deviation of the three sensors is particularly large, the fusion result deviation of the equal weight weighted fusion algorithm is relatively large, and the sensor with low accuracy will greatly affect the fusion performance. In this case, the unequal weight fusion algorithm will be used and the weight will be assigned according to the performance of the sensor. The better the performance of the sensor data, the greater the weight assigned, otherwise the smaller the value. The key of the algorithm is to solve the weight. The simulation results show that the weight solution proposed in this section is consistent with the state of sensor performance. In order to verify whether the algorithm can detect abnormal data in the sensor node data, the sensor node data mixed with abnormal data is used as experimental samples for verification. Experimental results show that the algorithm can effectively monitor abnormal data.

As shown in Fig. 9, reducing data traffic in wireless sensor networks is an important way to save energy. By processing the original row data into imprecise data and sending it to users, the data fusion technology greatly reduces the traffic in sensor networks, thus saving the energy of nodes and prolonging the network lifetime. Based on the prediction of data fusion technology in wireless sensor networks, a prediction model is established by using the historical data collected by nodes to achieve the purpose of reducing data transmission. The common method of this algorithm is: when the error between the predicted result and the actual collected value is less than the preset error

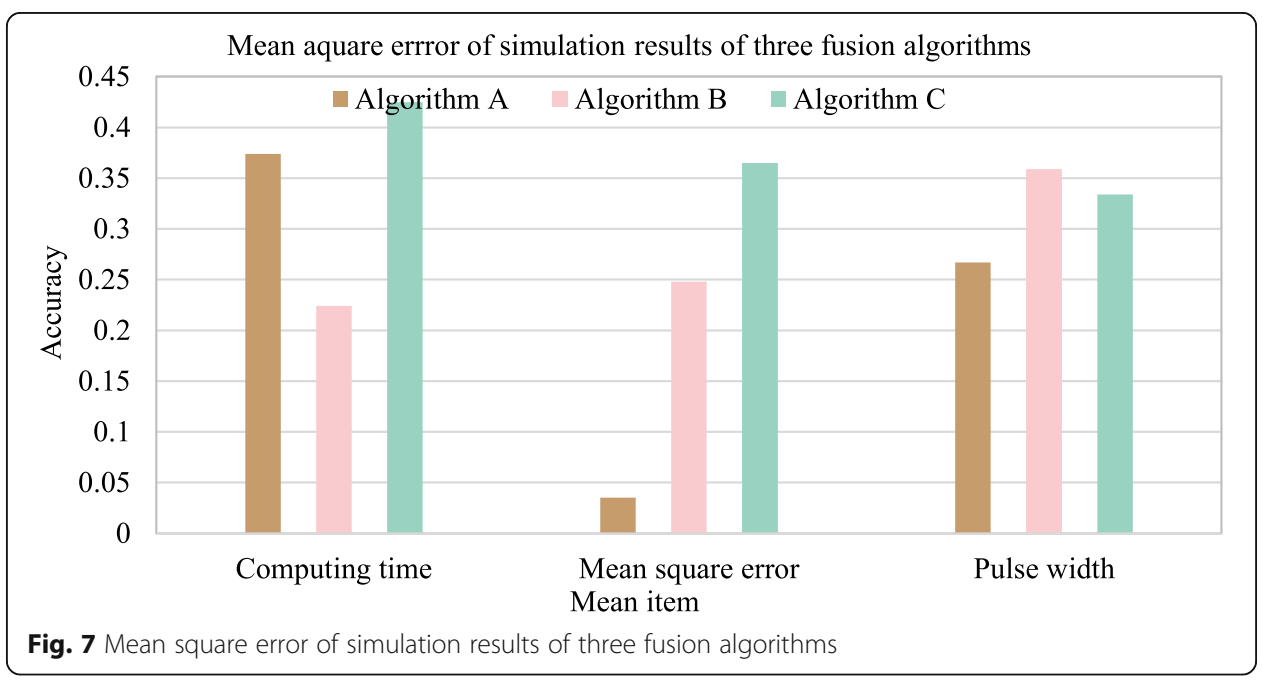




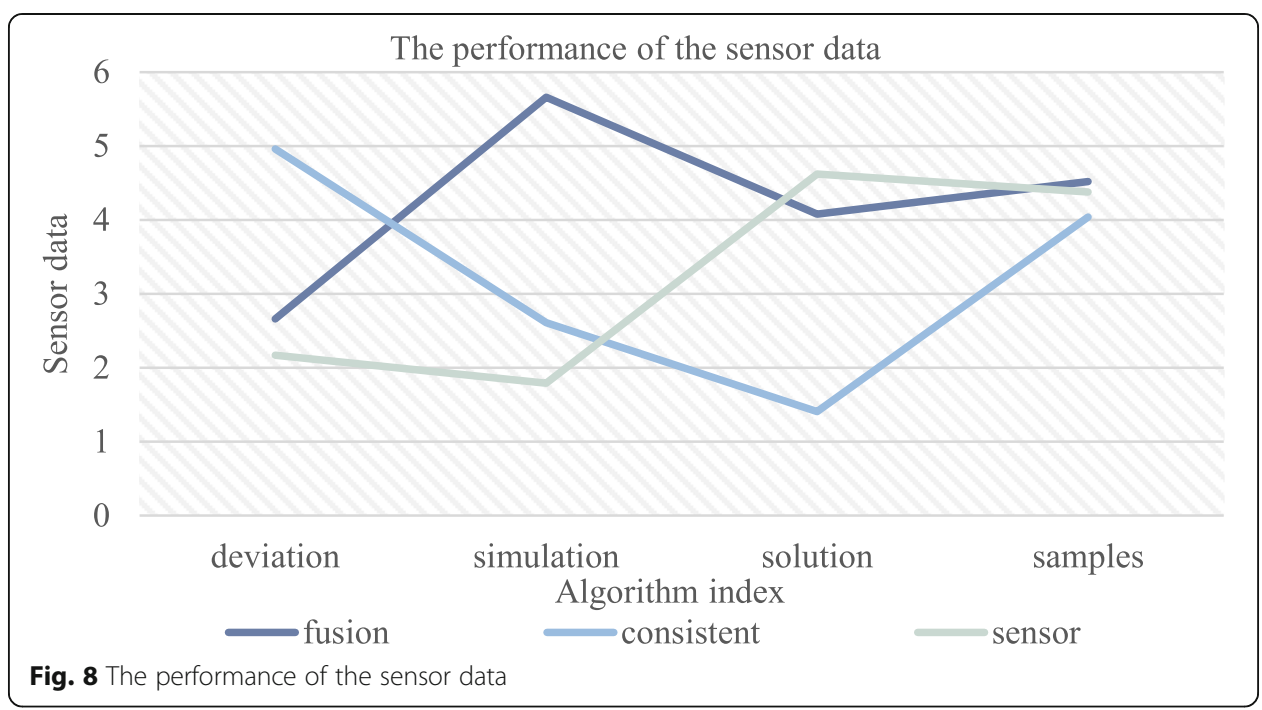

threshold, the source node does not need to send the collected value. Otherwise, the source node sends the collected actual values to the destination node and updates its prediction model.

As shown in Table 5, the prediction-based data fusion algorithm may have such a problem: when the node is affected by the environment or other human factors, the data obtained by the node itself is abnormal, which will lead to the prediction error greater than the error threshold. If the node sends the abnormal data to the host node according to the previous convention, it will not only affect the fusion result but also increase the network communication burden.

As shown in Fig. 10 and Table 6, in addition, due to the limited energy and the limited computing and storage capacity of sensor nodes, if the complexity of modeling algorithm or prediction algorithm is very high, there may be serious problems in practical operation. Therefore, it is necessary to reduce the complexity of prediction model and algorithm while ensuring the accuracy of prediction results.

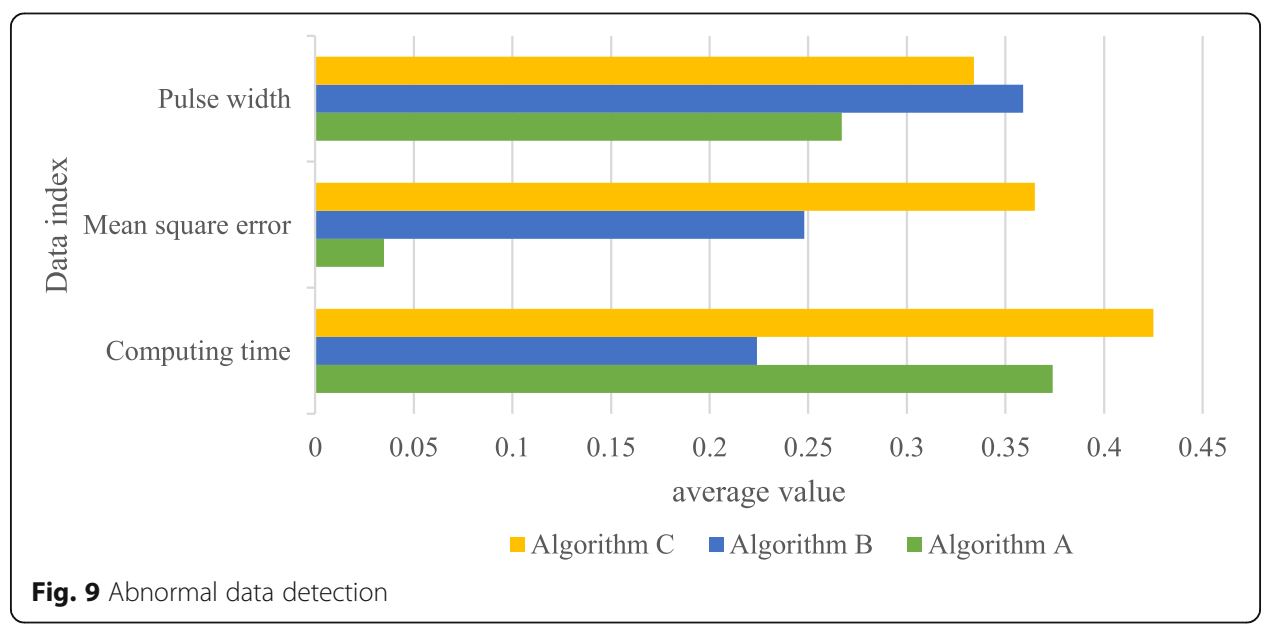


Table 5 The node sends the abnormal data to the host node

\begin{tabular}{llllll}
\hline Item & Fusion & Consistent & Sensor & Performance & Monitor \\
\hline Deviation & 2.66 & 4.96 & 2.17 & 2.32 & 4 \\
Simulation & 5.66 & 2.61 & 1.79 & 3.73 & 2.13 \\
Solution & 4.08 & 1.41 & 4.62 & 4.88 & 1.15 \\
Samples & 4.52 & 4.04 & 4.38 & 3.28 & 3.67 \\
\hline
\end{tabular}

\section{Conclusion}

Multi-sensor data fusion technology has made great progress in today's society. At the same time, the application of this technology has also played a role in the development of economic equipment. With the continuous improvement of people's living standards, people pay more and more attention to their own health conditions. I hope that multi-sensor data fusion technology can improve and improve people's health level. In this case, the information obtained by a single sensor is always imperfect. Therefore, multiple sensors are used to obtain more dimensional information about the target, ensure the integrity and accuracy of the information, and fuse the multidimensional information that can help us accurately. You need to detect the target from an angle to determine the physiological health of the user. This paper analyzes the multi-sensor information fusion technology in detail, explains the basic principles of multi-sensor information fusion, fusion process, fusion level, architecture, and general fusion algorithms, and describes the sensor information fusion technology and its biomedical. Build an application model for detection from application frameworks, control structures for multi-sensor integrated systems, information fusion, etc. The current situation of multi-sensor information fusion technology is studied in several aspects.

This paper summarizes the overall framework of multi-sensor information fusion, studies, and constructs a sensor information fusion technology and its application model in biomedical detection, multi rule information fusion architecture. The hierarchical fusion prediction model of feature level and decision level is realized. In the

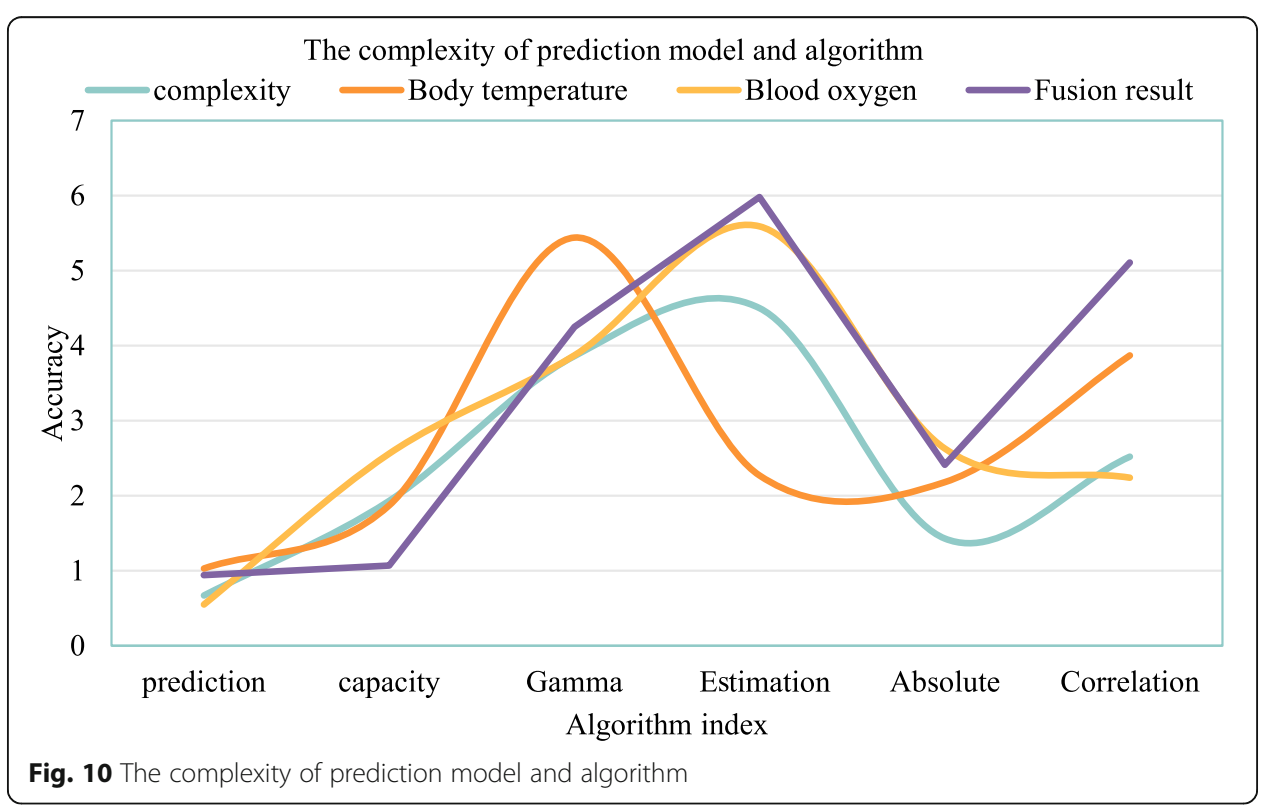


Table 6 The complexity of prediction model and algorithm

\begin{tabular}{lllll}
\hline Item & Complexity & Body temperature & Blood oxygen & Fusion result \\
\hline Prediction & 0.67 & 1.03 & 0.55 & 0.94 \\
Capacity & 1.93 & 1.87 & 2.56 & 1.07 \\
Gamma & 3.86 & 5.44 & 3.87 & 4.25 \\
Estimation & 4.5 & 2.27 & 5.59 & 5.98 \\
Absolute & 1.43 & 2.18 & 2.63 & 2.41 \\
Correlation & 2.52 & 3.87 & 2.24 & 5.11 \\
\hline
\end{tabular}

aspect of data fusion, several different data fusion calculation methods are used in the actual experiment.

In the past, there was a problem in the research of general data fusion algorithm. We need to manually set the upper limit of data fusion. The problem is that we usually set values based on experience. However, different values set by subjective experience lead to the difference or uncertainty of fusion results. Finally, the robustness and accuracy of the fusion results are affected. To solve the above problems, we propose a new algorithm. The theoretical basis of the algorithm is fuzzy theory. Based on this theory, multi-sensor data fusion is carried out. Firstly, the optimal fusion set of the algorithm is defined to obtain effective observation data. Then, the fusion degree matrix method is used to set the weight of different sensors reasonably, and simultaneous interpreting the estimation formula of data fusion. For the effectiveness of the algorithm, we can verify it through application examples or simulation, and the results are effective. Finally, due to the limited experience of the author, the research in this paper is not comprehensive and specific, and the author will continue to deepen the research in the future. The innovations of this article are: (1) Innovation and innovation in biomedical testing. (2) Use numerical values to characterize the degree of fusion in body temperature and blood oxygen fusion. (3) The performance of sensor data is increased at the biomedical level, showing the characteristics of multidisciplinary interaction.

Abbreviations

d-lof: Dynamic local outlier factor; WSN: Wireless sensor network; WMSN: Wireless medical sensor network; BSN: Because medical sensor network

Acknowledgements

Not applicable.

Author's contributions

Di Zhang has participated in conception and design, or analysis and interpretation of the data, drafting the article or revising it critically for important intellectual content. The author read and approved the final manuscript.

Funding

Not applicable

Availability of data and materials

Data sharing not applicable to this article as no datasets were generated or analyzed during the current study.

\section{Declarations}

Ethics approval and consent to participate

Not applicable.

Consent for publication

Approved. 


\section{Competing interests}

There is no potential conflict of interest in our paper and all authors have seen the manuscript and approved to submit to your journal. We confirm that the content of the manuscript has not been published or submitted for publication elsewhere.

Received: 19 May 2021 Accepted: 17 July 2021

Published online: 09 August 2021

\section{References}

1. Y. Yang, Q. Liu, Z. Gao, et al., Data fault detection in medical sensor networks. Sensors 15(3), 6066-6090 (2015)

2. H. Zhang, J. Liu, N. Kato, Threshold tuning-based wearable sensor fault detection for reliable medical monitoring using Bayesian network model. IEEE Syst. J. 12(2), 1886-1896 (2018)

3. D. He, N. Kumar, J. Chen, et al., Robust anonymous authentication protocol for health-care applications using wireless medical sensor networks. Multimedia Syst. 21(1), 49-60 (2015)

4. X. Li, J. Niu, S. Kumari, et al., A new authentication protocol for healthcare applications using wireless medical sensor networks with user anonymity. Secur. Commun. Netw. 9(15), 2643-2655 (2016)

5. Y. Zhang, L. Sun, H. Song and X. Cao, "Ubiquitous WSN for healthcare: recent advances and future prospects," in IEEE Internet of Things Journal, vol. 1, no. 4, pp. 311, 2014, doi: https://doi.org/10.1109/JIOT.2014.2329462.

6. A. Mishra, M. Mohapatro, An loT framework for bio-medical sensor data acquisition and machine learning for early detection. Int. J. Adv. Technol. Eng. Exploration 6(54), 112-125 (2019)

7. T. Kim, S. Kim, M. Cho, et al., TU-CD-304-09: feasibility study for thermoplastic mask set up monitoring using force sensing resistor (FSR) sensor. Med. Phys. 42(6), 3600-3601 (2015)

8. R. Mitchell, I.R. Chen, Network intrusion detection on cyber medical systems. IEEE Transact. Depend. Secur. Comput. 12(1), 16-30 (2015)

9. J. Yang, R. Qie, T. Li, et al., Nondestructive detection method of egg quality based on multi-sensor information fusion technology. J. Comput. Theor. Nanoence 13(9), 5932-5937 (2016)

10. Y. Maki, Psychological support for children and adolescents with gender dysphoria -from a clinical psychologist's viewpoint. Seishin shinkeigaku zasshi 119(1), 35-41 (2017)

11. Z. Zhang, X. Xiong, Research on the method of new generation of multi-sensor information fusion technology to promote the development of wisdom agriculture in 'internet + ' mode. Fresenius Environ. Bull. 25(12), 8030-8040 (2017)

12. C. Andonian, Beckmann, Jürgen, Biber $\mathrm{S}$, et al. Current research status on the psychological situation of adults with congenital heart disease. Cardiovasc. Diagn. Ther. 8(6), 799-804 (2018)

13. X. Ouyang, W. He, W. Lu, et al., Study on 3D border surveillance system and multi-sensor information fusion technology. Bandaoti Guangdian/Semiconductor Optoelectronics 39(2), 298-304 (2018)

14. R.G. Ma, Z.H. Ma, Y.X. Wang, Study on positioning technology of mileage piles based on multi-sensor information fusion. J. Highway Transport. Res. Dev. 10(4), 7-12 (2016)

15. B.L. Han, T. Zhang, Q.S. Luo, et al., Obstacle avoidance technology of bionic quadruped robot based on multi-sensor information fusion. J. Bjing Inst. Technol 25(4), 448-454 (2016)

16. M. Mosalanejad, M.M. Arefi, UKF-based soft sensor design for joint estimation of chemical processes with multi-sensor information fusion and infrequent measurements. ence. Meas. Technol. IET 12(6), 755-763 (2018)

17. M. Zhang, Z. Jiang, Reciprocating compressor fault diagnosis technology based on multi-source information fusion. Chinese J. Mech. Eng. 53(23), 1-8 (2017)

18. Y. Zhou, L. Chang, B. Qian, A belief-rule-based model for information fusion with insufficient multi-sensor data and domain knowledge using evolutionary algorithms with operator recommendations. Soft Comput. 23(13), 5129-5142 (2019)

19. H. Jin, T. Yang, X. Wang, et al., Application of multi-sensor information fusion in UAV relative navigation method. Guofang Keji Daxue Xuebao/J. Natl Univ. Def. Technol. 39(5), 90-95 (2017)

20. X. Gao, Y. Sun, D. You, et al., Multi-sensor information fusion for monitoring disk laser welding. Int. J. Adv. Manufact Technol. 85(5-8), 1167-1175 (2016)

21. J. Pang, J. Sun, Z. Zhang, Design and experimental study of fire warning system for data fusion. J. Information. Comput ence 12(9), 3629-3636 (2015)

22. P. Saha, S. Mukhopadhyay, Multispectral information fusion with reinforcement learning for object tracking in loT edge devices. IEEE Sens. J. 20(8), 4333-4344 (2020)

23. P.P. Armijo, M.R. León, C.C. Rodríguez. Instrument to assess educational programs for parents of children with congenital heart disease undergoing cardiac surgery. Arch. Argentinos De Pediatria 115(5), 439-445 (2017)

24. Z. Wanting, J. Yue, L. Shi, et al., Debris flow monitoring system design and experimental simulation of multiple information fusion based on electrical CT technology. J. Nat. Disasters 26(2), 185-195 (2017)

25. Y. Sun, H. Song, A.J. Jara, R. Bie, Internet of things and big data analytics for smart and connected communities. IEEE Access 4, 766-773 (2016). https://doi.org/10.1109/ACCESS.2016.2529723

26. F. Xiao, Multi-sensor data fusion based on the belief divergence measure of evidences and the belief entropy. Information Fusion 46, 23-32 (2019)

27. M. Zhou, X. Li, Y. Wang, S. Li, W. Nie, $6 \mathrm{~g}$ multi-source information fusion based indoor positioning via Gaussian kernel density estimation. (IEEE Internet of Things Journal, 2020) pp.1-1 (99). https://doi.org/10.1109/JIOT.2020.3031639

\section{Publisher's Note}

Springer Nature remains neutral with regard to jurisdictional claims in published maps and institutional affiliations. 